\title{
Integrative gene network and functional analyses identify a prognostically relevant key regulator of metastasis in Ewing sarcoma
}

\author{
Florencia Cidre-Aranaz 1,2, Jing Li, ${ }^{1,2}$, Tilman L. B. Hölting ${ }^{3}$, Martin F. Orth³ ${ }^{3}$ Roland Imle 1,4,5, \\ Stefanie Kutschmann ${ }^{1,2}$, Giulia Ammirati ${ }^{7}$, Katharina Ceranski ${ }^{1,2}$, Martha Julia Carreño-Gonzalez ${ }^{1,2}$, \\ Merve Kasan ${ }^{3}$, Aruna Marchetto ${ }^{3}$, Cornelius M. Funk ${ }^{1,2}$, Felix Bestvater ${ }^{8}$, Simone Bersini ${ }^{7}$, Chiara Arrigoni ${ }^{7}$, \\ Matteo Moretti ${ }^{7,9}$, Uwe Thiel ${ }^{10}$, Daniel Baumhoer ${ }^{11}$, Felix Sahm ${ }^{1,12,13}$, Stefan M. Pfister ${ }^{1,14}$, Wolfgang Hartmann ${ }^{15}$, \\ Uta Dirksen 16,17, Laura Romero-Pérez ${ }^{1,2}$, Ana Banito ${ }^{1,4}$, Shunya Ohmura ${ }^{1,2}$, Julian Musa ${ }^{1,2,18}$, Thomas Kirchner ${ }^{3}$, \\ Maximilian M. L. Knott ${ }^{3 \dagger}$ and Thomas G. P. Grünewald ${ }^{1,2,3,19^{*}+}$ (D)
}

The identification of cancer stemness genes is crucial to understand the underlying biology of therapy resistance, relapse, and metastasis. In pediatric tumors - despite being largely composed of undifferentiated stem celllike cells - mutations generally do not involve canonical stemness/metastasis-associated genes [1]. Ewing sarcoma (EwS), the second most common bone cancer in children and adolescents [2], is a highly aggressive malignancy associated with dismal outcome in metastatic disease [3], wherefore deciphering mechanisms of metastasis is imperative. EwS is characterized by a remarkably 'silent' genome with a single driver mutation generating an oncogenic fusion transcription factor (EWSR1-ETS) that promotes stem cell features $[4,5]$. Thus, EwS constitutes an ideal model to study how perturbation of a transcriptional network by a dominant oncogene can mediate metastasis.

To identify highly relevant EWSR1-ETS target genes involved in the stemness/metastasis axis we

\footnotetext{
*Correspondence: t.gruenewald@dkfz-heidelberg.de

${ }^{\dagger}$ Maximilian M. L. Knott and Thomas G. P. Grünewald contributed equally to this work.

2 Division of Translational Pediatric Sarcoma Research (B410), German Cancer Research Center (DKFZ), German Cancer Consortium (DKTK), Im Neuenheimer Feld 280, 69210 Heidelberg, Germany

Full list of author information is available at the end of the article
}

implemented a systems biology approach to analyze published transcriptome profiles of $5 \mathrm{EwS}$ cell lines with/without shRNA-mediated knockdown of EWSR1FLI1 or $-E R G(<20 \%)$ for $96 \mathrm{~h}[6]$ as described in Fig. 1a. This analysis yielded 348 differentially expressed genes (DEGs) being up- or downregulated $(|\log 2 \mathrm{FC}| \geq 1.5)$ after knockdown of EWSR1-FLI1 or -ERG across all cell lines (Supplementary Table 1). We next filtered these DEGs for genes annotated with the significantly enriched gene ontology term 'Regulation of Cell Differentiation' using PantherDB $\left(P=4.93 \times 10^{-12}\right.$, falsediscovery rate $=1.97 \times 10^{-9}$ ). The resulting $76 \mathrm{DEGs}$ (Supplementary Table 2), were subjected to network analysis for pathway, physical, and genetic interactions using Cytoscape and GeneMania (Fig. 1b, c). To identify clinically relevant key hubs within this network we focused on TFs $(n=11)$ that were highly interconnected ( $>10$ interactions) with a significant $(P<0.05$, MantelHaenszel test) association with overall survival in a cohort of $166 \mathrm{EwS}$ patients with matched gene expression and clinical data [7]. After adjustment for multiple testing (Supplementary Table 3), transcription factor 7 like 1 (TCF7L1, alias TCF3) emerged as the most promising candidate for functional follow-up, whose low expression was associated with poor patients' overall survival (nominal $P=0.0057 ; P=0.0228$ Bonferroni-adjusted) original author(s) and the source, provide a link to the Creative Commons licence, and indicate if changes were made. The images or other third party material in this article are included in the article's Creative Commons licence, unless indicated otherwise in a credit line to the material. If material is not included in the article's Creative Commons licence and your intended use is not permitted by statutory regulation or exceeds the permitted use, you will need to obtain permission directly from the copyright holder. To view a copy of this licence, visit http://creativecommons.org/licenses/by/4.0/. The Creative Commons Public Domain Dedication waiver (http://creativecommons.org/publicdomain/zero/1.0/) applies to the data made available in this article, unless otherwise stated in a credit line to the data. 
(Fig. 1d). Next, we explored TCF7L1 protein levels in association with clinical outcome in tissue micro-arrays (TMAs) comprising 114 EwS tumors and found that low TCF7L1 protein expression was significantly $(P=0.035)$ associated with worse overall survival (Fig. 1e), supporting that low TCF7L1 upregulation of TCF7L1 (Supplementary Table 4), which was confirmed in $5 \mathrm{EwS}$ cell lines by qRT-PCR (average $\sim 6$-fold upregulation) (Supplementary Fig. 1b) and validated in A673 cells xenografts with/without conditional knockdown of EWSR1-FLI1 (A673/TR/shEF1 cells) at mRNA and protein levels (Supplementary Fig. 1c).

Prior reports suggested that TCF7L1 may have either oncogenic or tumor suppressor properties depending on the cellular context. Indeed, TCF7L1 expression has been linked to promotion of cell proliferation, tumor growth and sphere formation in breast cancer [8], colorectal cancer $[9,10]$, acute lymphoblastic leukemia [11] and skin squamous cell carcinoma [12], while ectopic expression of TCF7L1 inhibits self-renewal of liver cancer stem cells [13]. To explore its role in EwS, we first analyzed the TCF7L1 expression pattern across 18 cancer entities using microarray data from the Cancer Cell Line Encyclopedia (CCLE) and an own study that compiled well-curated microarray data from the same cancer entities [14]. Surprisingly,
TCF7L1 was very highly, but variably, expressed in EwS cell lines and primary tumors (Fig. 1f).

Weighted Gene Correlation Network Analysis (WGCNA) based on enriched gene-sets in TCF7L1-correlated genes in $166 \mathrm{EwS}$ tumors showed that EwS tumors with low TCF7L1 expression were enriched in embryonic pathways (Fig. 1g). Although TCF7L1 is generally highly expressed in EwS, these data suggested that suppression of its transcription by EWSR1-FLI1 is associated with embryonic processes that may be linked with poor patient outcome. Thus, we generated two EwS cell lines (SK-NMC and TC-71, with lowest TCF7L1 expression across the 5 EwS cell lines tested (Supplementary Fig. 1a) with a doxycycline- (DOX)-inducible re-expression of TCF7L1 (Fig. 1h). Consistent with the hypothesis that TCF7L1 may be a EWSR1-ETS-repressed downstream transcription factor (with a potentially indirect regulation, possibly partially mediated by TCF7L1 promoter hypermethylation as depicted in Supplementary Fig. 1d-g), transcriptome profiling of two EwS cell lines after either knockdown of EWSR1-ETS or re-expression of TCF7L1 showed a highly significant $\left(P=2.57 \times 10^{-165}\right.$ or $\left.P=5.34 \times 10^{-272}\right)$ overlap of concordant DEGs (Supplementary Fig. 1e). Congruently, conditional re-expression of TCF7L1 significantly reduced cell proliferation (Fig. 1i), clonogenic

\footnotetext{
(See figure on next page.)

Fig. 1 Systems biology identifies TCF7L1 as a prognostically relevant EWSR1-ETS-regulated network hub whose re-expression inhibits tumorigenesis in vitro and in vivo. a) Workflow depicting a systems biology approach to identify DEGs regulated by EWSR1-ETS, involved in regulation of cell differentiation, functioning as highly interconnected TFs, and associated with overall survival in a cohort of 166 EwS patients. Number of genes represent remaining candidates after each filtering step. b) Network of EWSR1-ETS-regulated genes involved in regulation of cell differentiation. Genes are depicted as nodes (circles). Blue nodes represent TFs, node outline color show up- (green) or down- (red) regulation by EWSR1-ETS. Node border width represent strength of regulation by EWSR1-ETS (the thicker the border the higher the fold-change). Black dots surrounding the nodes represent interconnections with other nodes within the network. Connecting lines show three types of interconnection: physical (red), pathway (blue), genetic (brown). Line width represents strength of interconnection. c) Close-up image of highly interconnected TFs located at the center of the network (blue nodes). d) Kaplan-Meier survival analysis of 166 primary EwS patients stratified by quintile TCF7L1 expression. Mantel-Haenszel test, Bonferroni corrected. DEG: differentially expressed genes; KD: knockdown. e) Kaplan-Meier survival analysis of 114 EwS patients stratified by TCF7L1 protein expression intensity (low intensity $\leq 1$, high intensity $>1$ ). $P$-value was determined by Gehan-Creslow-Wilcoxon test. Representative micrographs of TCF7L1 IHC are depicted. Scale bar $=50 \mu \mathrm{m}$. f) TCF7L1 expression in cell lines or primary tumors from EwS and 17 additional tumor entities (Cancer Cell Line Encyclopedia, CCLE). Data are represented as bar plots where horizontal bars represent mean and SEM. The number of samples per group $(n)$ is given in parentheses. RMS, rhabdomyosarcoma; NSCLC, non-small cell lung carcinoma; SCLC: small-cell lung carcinoma. g) Weighted Gene Correlation Network Analysis (WGCNA) of enriched gene-sets obtained by Pearson correlation analysis of genes whose expression is negatively correlated with TCF7L1 expression in Affymetrix expression data of 166 EwS tumors. Network depicts signatures presenting NES $>1.5$ and $P<0,05$. NES, normalized enrichment score. h) Relative TCF7L1 expression (qRT-PCR) of TC-71 and SK-N-MC cells containing a DOX-inducible re-expression construct for TCF7L1. Cells were grown either with or without DOX for $72 \mathrm{~h} . n \geq 6$ biologically independent experiments. Two-sided Mann-Whitney test. i) Viable cell count of TC-71 and SK-N-MC cells containing a DOX-inducible TCF $1 \mathrm{~L} 1$ re-expression construct $72 \mathrm{~h}$ after DOX-treatment. Data are mean and SEM, $n=7$ biologically independent experiments. Two-sided Mann-Whitney test. j) Relative area covered by colonies (\%) grown in soft-agar of EwS cells containing a DOX-inducible re-expression construct for TCF7L1. Cells were grown either with/without DOX-treatment. $n=3$ biologically independent experiments. Two-sided Mann-Whitney test. k) Growth of subcutaneous xenografts of indicated EwS cells containing a DOX-inducible TCF7L 1 re-expression construct (arrow $=$ start of DOX-treatment). Data are represented as means ( $n=7$ animals/group). Two-sided Mann-Whitney test. I) Ex vivo analysis of relative necrotic area (top) and mitotic index (bottom) of xenografted TC-71 and SK-N-MC cell lines. Data are mean and SEM, $n=7$ animals/group. $\mathbf{m}$ ) Ex vivo analysis of Ki67 positivity of xenografted TC-71 and SK-N-MC cell lines. Horizontal bars represent means and whiskers SEM, $n=7$ animals/group. $P$-values were determined via $x^{2}$ test testing all positives (high and moderate immunoreactivity) versus negatives. Histological images depict representative Ki67 micrographs. Scale bar $=50 \mu \mathrm{m}$.
} 


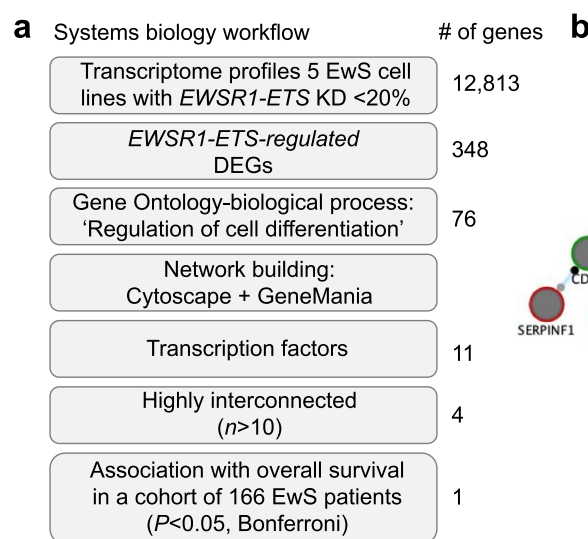

e
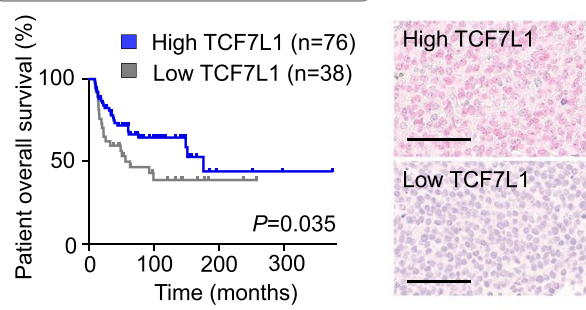

f
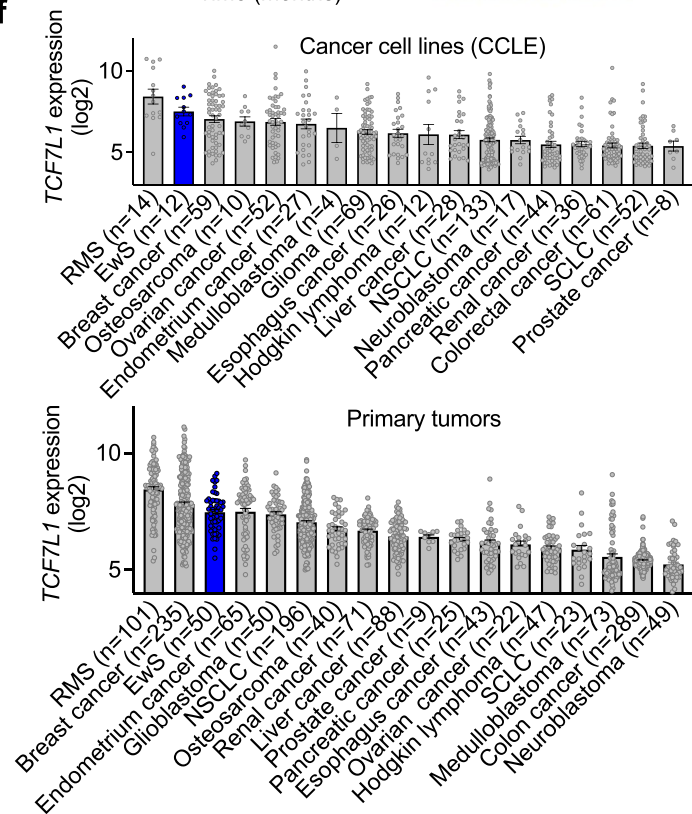

g TCF7L1-correlated signatures in 166 primary EwS

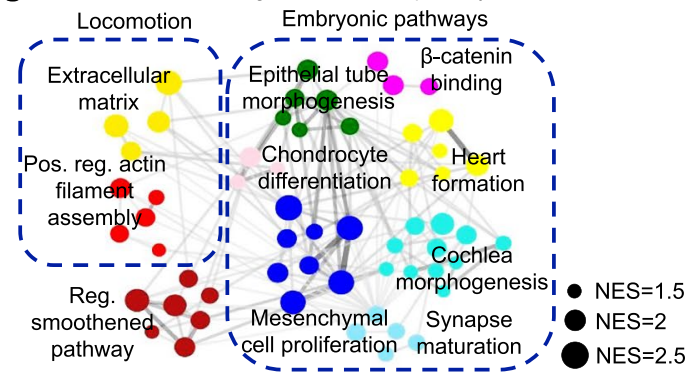

Fig. 1 (See legend on previous page.) c
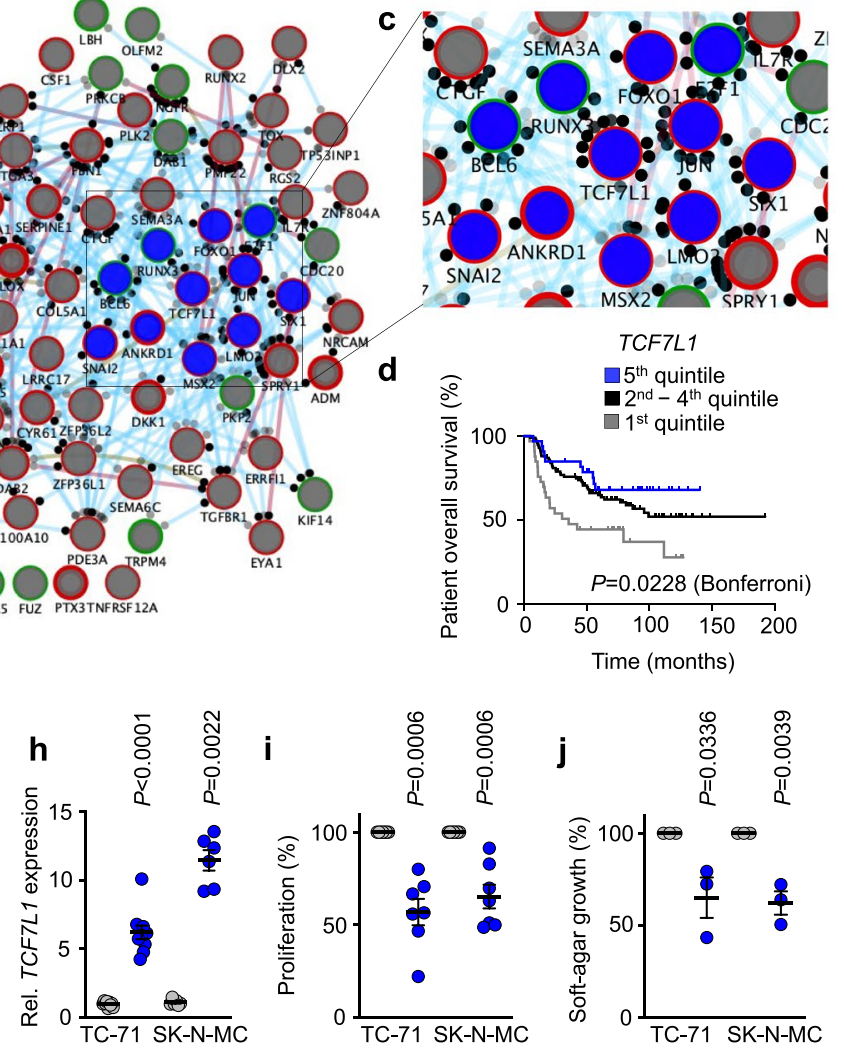

k

$\square \operatorname{DOX}(-)$
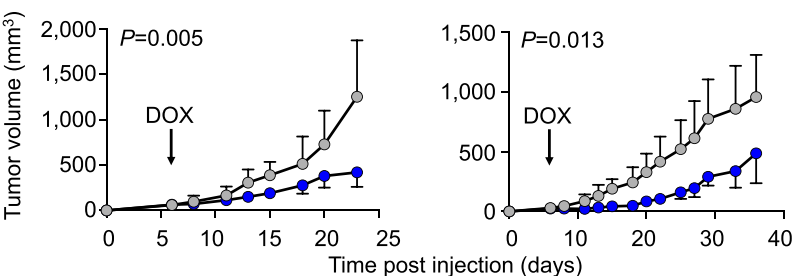

m
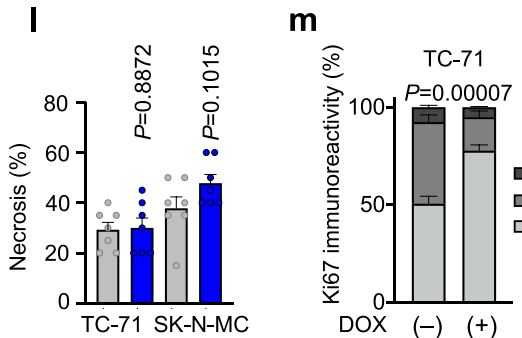

SK-N-MC

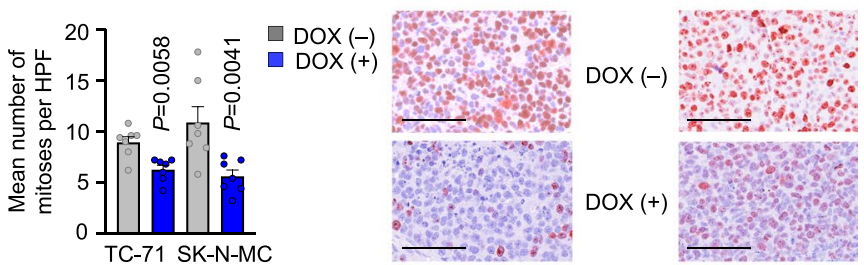


growth in two-dimensional (2D) cultures (Supplementary Fig. 2b), anchorage-independent and three-dimensional (3D) growth (Fig. 1j), and local tumor growth in a preclinical subcutaneous xenotransplantation mouse model (Fig. 1k). The xenografts exhibiting specific re-expression of TCF7L1 (DOX $(+)$ group) (Supplementary Fig. 2e,f) showed a reduced mitotic index (Fig. 1l), and a significant decrease of the proliferation marker Ki67 (Fig. 1m). Similar in vitro and in vivo experiments performed with both cell lines 'empty' controls exhibited no significant differences (Supplementary Fig. 2a, c-d, g-i).

Since stemness features, such as elevated clonogenic capacity and anchorage-independent growth, are essential for circulating tumor cells to colonize and develop into clinically apparent metastases in distant organs, we reasoned that TCF7L1 may be linked to the metastatic process in EwS. In support of this hypothesis, transcriptome profiling, subsequent gene-set enrichment and WGCNA of TCF7L1-reexpressing SK-N-MC and TC-71 EwS cells uncovered that low TCF7L1 levels led to overrepresentation of gene-sets involved in cellular migration (Fig. 2a). Accordingly, re-expression of TCF7L1 resulted in significantly reduced transwell migration (Fig. 2b), invasion, and single-cell 3D migration in fibrin gel using advanced microfluidic chambers (Fig. 2c). Next, we employed an orthotopic spontaneous in vivo metastasis model by injecting TCF7L1 re-expressing cells into the proximal tibiae of immunocompromised mice. Remarkably, while there was no significant difference in local tumor growth in the limited space of the tibial plateau (Supplementary
Fig. 3a,b), we observed a strong inhibition of macrometastatic spread to liver, lungs, and kidneys upon re-expression of TCF7L1, and significantly reduced micrometastatic burden in the same organs (Fig. 2d,e, Supplementary Fig. $3 \mathrm{c}-\mathrm{e})$. In agreement with these in vivo findings, we observed that TCF7L1 was significantly $(P=0.016)$ lower expressed in $\mathrm{EwS}$ metastases $(n=7)$ compared to primary tumors $(n=118)$ in situ as defined by RNA-sequencing of tumor tissue (Fig. 2f). Strikingly, this result became even more significant when focusing on gene expression data of 4 matched pairs of metastases and primaries $(P=0.0078)$ (Fig. 2g).

To further investigate the mechanism of action of TCF7L1, we generated two EwS cell lines with conditional re-expression of different TCF7L1 deletion mutants for its major domains ( $\triangle$ HMG: DNA binding domain; $\triangle$ CTNNB: $\beta$-catenin binding domain) and observed that only the $\triangle$ HMG mutants exhibited a normal clonogenic growth and migration capacity in vitro (Fig. $2 \mathrm{~h}, \mathrm{i}$ ), and tumor growth in vivo (Fig. 2j). Moreover, further analysis of our WCGNA on TCF7L1-regulated signatures displayed in Fig. 2a highlighted several downregulated 'driver target genes' (aka leading-edge genes), including ANXA1, LMO7, SLC9A9, and TMEM71 as potential key mediators of TCF7L1 inhibition of the EwS migratory phenotype. These genes were selected for validation as they ranged among the top 10 downregulated genes by TCF7L1 and as their upregulation has been previously implicated in cancer progression [15-18]. As shown in Supplementary Fig. 3f, these genes are strongly downregulated upon

\footnotetext{
(See figure on next page.)

Fig. 2 High TCF7L1 expression inhibits metastasis in EwS through its DNA binding domain. a) Weighted Gene Correlation Network Analysis (WGCNA) depicting functional gene enrichment of down- or up-regulated genes in TCF7L1 re-expressing EwS cells. Network depicts signatures presenting $P<0,05, N E S>2$. NES, normalized enrichment score. Arrows depict direction of gene regulation. b) Relative percentage of migrated cells in $6 \mathrm{~h}$. TC-71 and SK-N-MC EwS cells containing a DOX-inducible re-expression construct for TCF7L1 where pre-treated with or without DOX for $72 \mathrm{~h} . n \geq 7$ biologically independent experiments. Two-sided Mann-Whitney test. c) Invasion and single cell migrated distance in $15 \mathrm{~h}$. TC-71 and SK-N-MC EwS cells containing a DOX-inducible re-expression construct for TCF7L 1 where pre-treated with or without DOX for $72 \mathrm{~h}$ and added to a microfluidic chamber containing a fibrin compartment. $n \geq 3$ biologically independent experiments. Two-sided unpaired t-test. d) Schematic representation of the experimental design: TC-71 or SK-N-MC EwS cell lines containing a DOX-inducible re-expression construct for TCF $L 1$ were injected in the right tibia plateau. Animals were subsequently randomized and treated with or without DOX. At the end of the experiment, mice were evaluated ex vivo for presence of spontaneous metastases in inner organs. Pie charts depict percentage of metastasis-free organs (blue) in each condition, $n$ represents total number of metastasis. Bottom pictures show representative images of metastatic organs in DOX ( - ) and DOX (+) conditions. $n=8$ animals/group. e) Graph depicts relative area of histological metastatic spread of orthotopically injected SK-N-MC EwS cells containing a DOX-inducible re-expression construct for TCF7L1. $n=21$ slides/group. Two-sided Mann Whitney test. Pictures show representative histological images of HE stainings from the evaluated organs. Scale bar is $50 \mu \mathrm{m}$. M, metastasis; N, normal tissue. f) Comparison of relative TCF7L 1 expression of EWS primary tumors versus metastasis $(n=125)$, normalized using RPLPO expression of the same samples. $\mathbf{g}$ ) Analysis of relative TCF7L1 expression (normalized to RPLPO expression of the same samples) in paired metastasis/primary samples from EwS patients 1-4. Independent one sample t-test. h) Relative colony number of colony-forming assays (CFAs) of TC-71 (left) and SK-N-MC (right) cells containing a DOX-inducible re-expression construct for TCF7L1, empty control, or one of the two deletion mutants for TCF7L1 (deletion mutant for the $\beta$-catenin binding domain, $\triangle C T N N B$; deletion mutant for the DNA binding domain, $\triangle H M G$ ). Cells were grown either with or without DOX. $n=4$ biologically independent experiments. i) Relative percentage of migrated cells in $6 \mathrm{~h}$. TC-71 and SK-N-MC EwS cells containing a DOX-inducible re-expression construct for TCF7L1 DNA binding domain ( $\triangle \mathrm{HMG}$ ) where pre-treated with or without DOX for $72 \mathrm{~h} . n \geq 8$ biologically independent experiments. j) Growth of EwS subcutaneous xenografts of TC-71 and SK-N-MC cells containing a DOX-inducible re-expression construct for TCF7L1 deletion mutant $\triangle H M G$ (arrow indicates start of DOX treatment). Data are represented as means ( $n=7$ animals/group). Two-sided Mann-Whitney test
} 

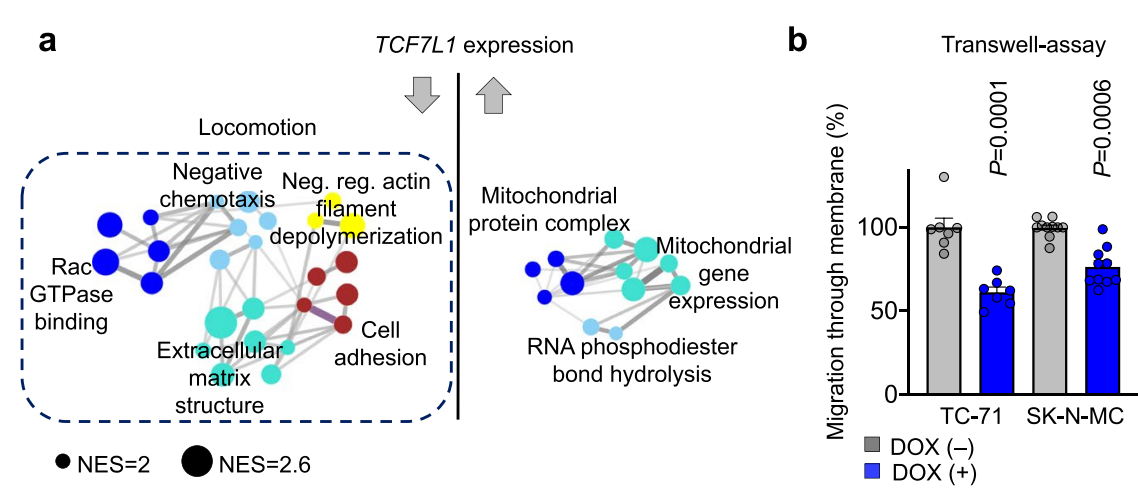

C Single-cell $3 D$ tracing assay

d

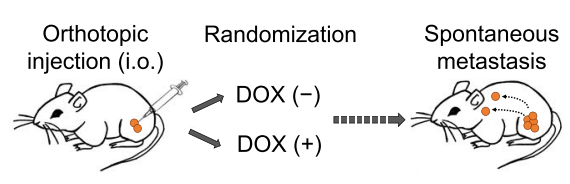

Percentage of

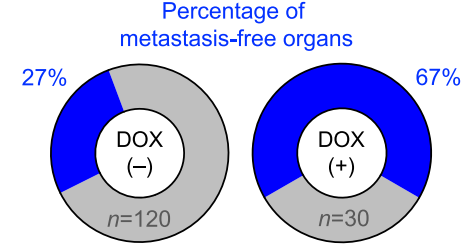

Total number of macro-metastases across organs

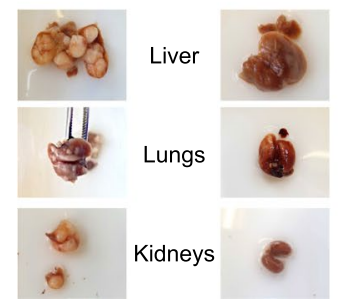

h

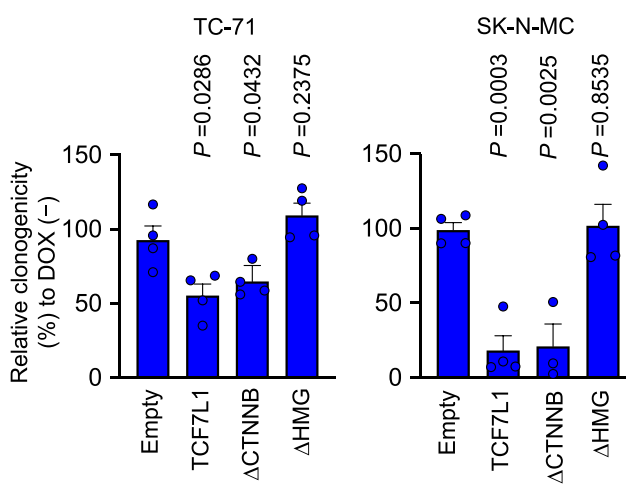

e
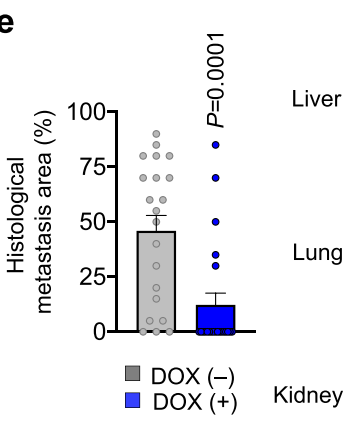

f

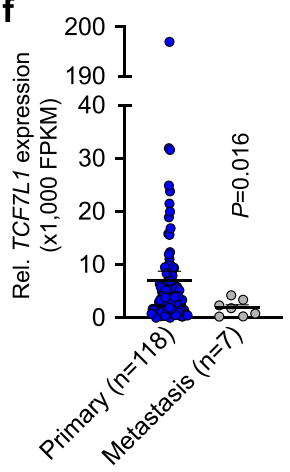

i

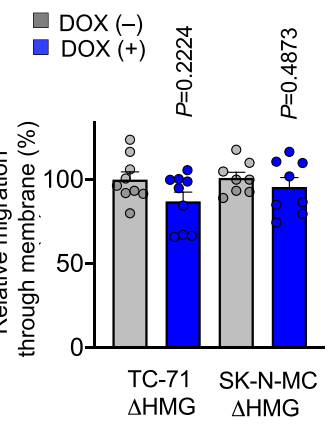

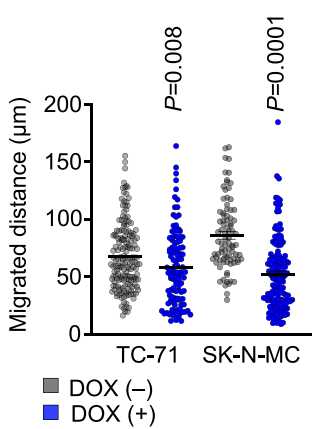

$\operatorname{DOX}(-)$

$\operatorname{DOX}(+)$

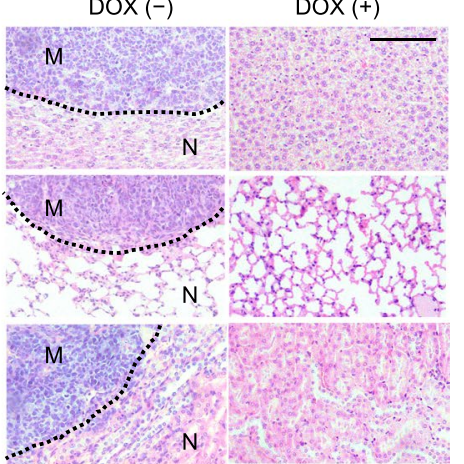

g

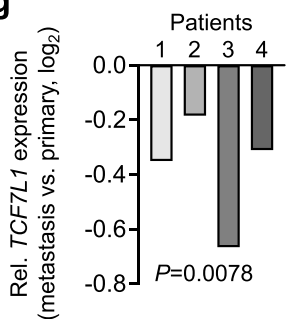

j

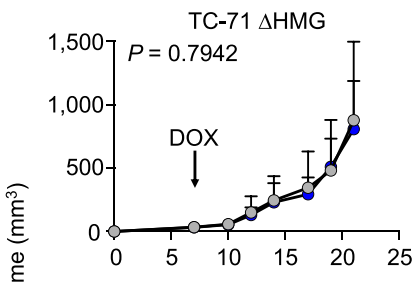

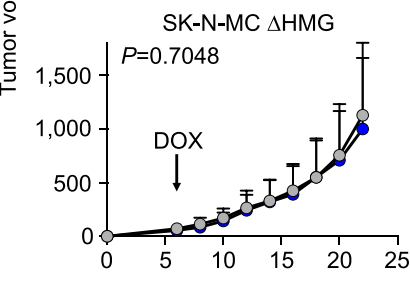

Time post injection (days)

Fig. 2 (See legend on previous page.) 
re-expression of wildtype TCF7L1 in both EwS cell lines tested, but remain unaltered in the DNA-binding-domain deletion mutant $(\triangle \mathrm{HMG})$, further suggesting that DNAbinding of TCF7L1 is required to suppress the tumorigenic and migratory phenotype of EwS cells.

\section{Conclusions}

Previous studies proposed a binary model of EWSR1FLI1 ${ }^{\text {-high }}$ promoting proliferation and EWSR1-FLI1 ${ }^{\text {-low }}$ promoting metastasis [19] following a traditional epithelial-mesenchymal-transition concept. However, our findings support an integrative concept of the 'migratory stem cell' [20]: EwS cells may reside in a 'metastable' state where all EwS cells of a tumor may be equipped with both proliferative and migratory capacities [21]. Upon different intrinsic/extrinsic cues (of which EWSR1-FLI1 may not be the only important component) these cells could underlie a certain accentuation.

In this context, we demonstrated that TCF7L1 is a critical mediator of metastasis in EwS, which may be utilized as a prognostic biomarker. Since we could detect a strong inverse correlation of TCF7L1 levels with patients' overall survival in both the mRNA- as well as in the TMA-cohort, we believe that the most straightforward possibility to translate TCF7L1 into a routine clinical setting would be the IHC detection and semiquantitative evaluation of TCF7L1 in primary biopsies. This technique is readily available, inexpensive, and the conditions of IHC staining of TCF7L1 in EwS tumors have been established in the current study, which would offer the possibility of further evaluating the prognostic value of TCF7L1 in ongoing and prospective clinical trials. In addition, it would be highly interesting to explore whether TCF7L1 may serve as a prognostic biomarker in other (EWSR1-rearranged) mesenchymal neoplasms, which is subject to future studies.

In summary, our study exemplifies the power of systems biology to decipher gene regulatory networks and to identify key players in the metastatic process, which may be highly relevant for, and translatable to, other oligomutated (pediatric) cancers.

\footnotetext{
Abbreviations

CCLE: Cancer Cell Line Encyclopedia; CTNNB: Beta catenin; DOX: Doxycycline; DEG: Differentially expressed genes; EMT: Epithelial to mesenchymal transition; ETS: E26 transformation specific; EwS: Ewing sarcoma; EWSR1: Ewing sarcoma breakpoint region 1; ERG: ETS transcription factor ERG; FDR: False discovery rate; FET: FUS, EWSR1, TAF15; FC: Fold change; FFPE: Formalin-fixed and paraffin-embedded; FLI1: Friend leukemia virus integration 1; GO: Gene ontology; GSEA: Gene set enrichment analysis; HE: Hematoxylin Eosin; HMG: High mobility group; IHC: Immunohistochemistry; IRS: Immune reactive score; MTT: 3-[4,5-dimethylthiazol-2-yl]-2,5 diphenyl tetrazolium bromide; NES: Normalized enrichment score; NSG: NOD/SCID/gamma; TCF3: T-cell factor 3; TCF7L1: Transcription factor 7 like 1; TF: Transcription factor; TMA: Tissue microarray; ORF: Open reading frame; WGCNA: Weighted gene co-expression network analysis; Wnt:Wingless/Integrated
}

\section{Supplementary Information}

The online version contains supplementary material available at https://doi. org/10.1186/s12943-021-01470-z.

\section{Additional file 1:}

Additional file 2:

\section{Additional file 3:}

\section{Acknowledgements}

We thank Dr. Paul Northcott and Dr. Brian Gudenas for providing R scripts for weighted gene correlation network analyses and Carlos Rodriguez-Martin for assistance with script adaptation. We thank Susanne Jabar and Dr. Andreas Ranft for statistical advice. We thank Anja Heier, Andrea Sendelhofert, Lea Hofmann and Laura Dörner for expert technical assistance. We thank Dr. Martin Sill for contributing to methylation analyses.

\section{Authors' contributions}

F.C.A, M.M.L.K., and T.G.P.G. conceived the study. F.C.A. and T.G.P.G wrote the paper, and drafted the figures and tables F.C.A. and M.M.L.K. carried out in vitro experiments. F.C.A, M.F.O., and T.G.P.G. performed bioinformatic and statistical analyses. F.C.A, M.M.L.K., J.L., T.L.B.H., J.M, R.I, and A.B. performed and/or coordinated in vivo experiments. S.K., M.F.O., K.C., M.J.C.G., G.A., S.B., C.A., M.M., C.F., A.M., S.O., L.R.P., and M.K. contributed to experimental procedures. W.H., D.B. and U.T. provided clinical and/or statistical guidance. T.K., F.S., S.M.P., and F.B. provided laboratory infrastructure, contributed to RNA sequencing and/or provided histological guidance. T.G.P.G. supervised the study and data analysis. All authors read and approved the final manuscript.

\section{Funding}

This work was mainly supported by the Wilhelm Sander-Foundation (2016.167.1), the Barbara \& Hubertus Trettner Foundation, and the Dr. Rolf M. Schwiete Foundation. In addition, the laboratory of T.G.P.G. was supported by the Matthias-Lackas Foundation, the Dr. Leopold and Carmen Ellinger Foundation, the German Cancer Aid (DKH-70112257 and DKH-70114111), the Gert und Susanna Mayer Foundation, the SMARCB1 association, and the Barbara und Wilfried Mohr Foundation. J.L. was supported by a scholarship of the Chinese Scholarship Council (CSC), and M.K. and C.M.F. by scholarships from the German Cancer Aid. M.M. was supported by the Swiss National Science Foundation (SNF 310030_179167). U.T. was supported by the Wilhelm SanderFoundation (2021.085.1), the Dr. Sepp and Hanne Sturm Foundation and by Zukunft Gesundheit e.V.

\section{Availability of data and materials}

Original microarray data that support the findings of this study were deposited at the National Center for Biotechnology Information (NCBI) GEO and are accessible through the accession number GSE165929. Custom code is available from the corresponding author upon reasonable request.

\section{Declarations}

\section{Ethics approval and consent to participate}

Human tissue samples were retrieved from the tissue archives of the Institute of Pathology of the LMU Munich (Germany), the Bone Tumor Reference Center at the Institute of Pathology of the University Hospital of Basel, or the GerhardDomagk Institute of Pathology of the University of Münster (Germany) upon approval of the institutional review board. All patients provided informed consent. Tissue-microarrays (TMAs) were stained and analyzed with approval of the ethics committee of the LMU Munich (approval no. 550-16 UE).

Animal experiments were approved by the government of Upper Bavaria and Northbaden and performed in accordance with ARRIVE guidelines, recommendations of the European Community (86/609/EEC), and United Kingdom Coordinating Committee on Cancer Research (UKCCCR) guidelines for the welfare and use of animals in cancer research.

Consent for publication

Not applicable. 


\section{Competing interests}

T.G.P.G. serves as honorary consultant for Boehringer-Ingelheim International $\mathrm{GmbH}$. All other authors declare no conflict of interest.

\section{Author details}

${ }^{1}$ Hopp-Children's Cancer Center (KiTZ), Heidelberg, Germany. ${ }^{2}$ Division of Translational Pediatric Sarcoma Research (B410), German Cancer Research Center (DKFZ), German Cancer Consortium (DKTK), Im Neuenheimer Feld 280, 69210 Heidelberg, Germany. ${ }^{3}$ Max-Eder Research Group for Pediatric Sarcoma Biology, Institute of Pathology, Faculty of Medicine, LMU Munich, Munich, Germany. ${ }^{4}$ Soft-Tissue Sarcoma Junior Research Group, German Cancer Research Center (DKFZ), German Cancer Consortium (DKTK), Heidelberg, Germany. ${ }^{5}$ Faculty of Biosciences, Heidelberg University, Heidelberg, Germany. ${ }^{6}$ Division of Pediatric Surgery, Department of General, Visceral and Transplantation Surgery, University Hospital Heidelberg, Heidelberg, Germany. ${ }^{7}$ Regenerative Medicine Technologies Laboratory, Ente Ospedaliero Cantonale (EOC), Lugano, Switzerland. ${ }^{8}$ Light Microscopy Facility (W210), German Cancer Research Center (DKFZ), German Cancer Consortium (DKTK), Heidelberg, Germany. ${ }^{9}$ Biomedical Sciences Faculty, Università della Svizzera Italiana (USI), Lugano, Switzerland. ${ }^{10}$ Technical University of Munich, School of Medicine, Department of Pediatrics and Children's Cancer Research Center, Munich, Germany. ${ }^{11}$ Bone Tumor Reference Center, Institute of Pathology of the University Hospital of Basel, Basel, Switzerland. ${ }^{12}$ Clinical Cooperation Unit (CCU) Neuropathology, German Cancer Research Center (DKFZ), German Cancer Consortium (DKTK), Heidelberg, Germany. ${ }^{13}$ Department of Neuropathology, Institute of Pathology, Heidelberg University Hospital, Heidelberg, Germany. ${ }^{14}$ Division of Pediatric Neuro-Oncology, German Cancer Research Center (DKFZ), German Cancer Consortium (DKTK), Heidelberg, Germany. ${ }^{15}$ Division of Translational Pathology, Gerhard-Domagk-Institute for Pathology, University Hospital Münster, Münster, Germany. ${ }^{16}$ Pediatrics III, AYA Unit, West German Cancer Centre, University Hospital Essen, Essen, Germany. ${ }^{17}$ German Cancer Consortium (DKTK), partner site Essen, Essen, Germany. ${ }^{18}$ Department of General, Visceral and Transplantation Surgery, Heidelberg University Hospital, Heidelberg, Germany. ${ }^{19}$ Institute of Pathology, Heidelberg University Hospital, Heidelberg, Germany.

Received: 3 September 2021 Accepted: 24 November 2021

Published online: 03 January 2022

\section{References}

1. Lawrence MS, Stojanov P, Polak P, Kryukov GV, Cibulskis K, Sivachenko A, et al. Mutational heterogeneity in cancer and the search for new cancerassociated genes. Nature. 2013;499(7457):214-8.

2. Grünewald TGP, Cidre-Aranaz F, Surdez D, Tomazou EM, de Álava E, Kovar $\mathrm{H}$, et al. Ewing sarcoma. Nat Rev Dis Primer. 2018 05;4(1):5.

3. Thiel U, Wawer A, Wolf P, Badoglio M, Santucci A, Klingebiel T, et al. No improvement of survival with reduced- versus high-intensity conditioning for allogeneic stem cell transplants in Ewing tumor patients. Ann Oncol Off J Eur Soc Med Oncol. 2011;22(7):1614-21.

4. Tirode F, Laud-Duval K, Prieur A, Delorme B, Charbord P, Delattre O. Mesenchymal stem cell features of Ewing tumors. Cancer Cell. 2007; 11(5):421-9.

5. Riggi N, Suvà M-L, De Vito C, Provero P, Stehle J-C, Baumer K, et al. EWSFLI-1 modulates miRNA145 and SOX2 expression to initiate mesenchymal stem cell reprogramming toward Ewing sarcoma cancer stem cells. Genes Dev. 2010;24(9):916-32.

6. Orth MF, Surdez D, Marchetto A, Grossetête S, Gerke JS, Zaidi S, et al. Systematic multi-omics cell line profiling uncovers principles of Ewing sarcoma fusion oncogene-mediated gene regulation [Internet]. 2021 Jun [cited 2021 Aug 16] p. 2021.06.08.447518. Available from: https://www. biorxiv.org/content/10.1101/2021.06.08.447518v1

7. Musa J, Cidre-Aranaz F, Aynaud M-M, Orth MF, Knott MML, Mirabeau O, et al. Cooperation of cancer drivers with regulatory germline variants shapes clinical outcomes. Nat Commun. 2019 11;10(1):4128.

8. Slyper M, Shahar A, Bar-Ziv A, Granit RZ, Hamburger T, Maly B, et al. Control of breast cancer growth and initiation by the stem cell-associated transcription factor TCF3. Cancer Res. 2012;72(21):5613-24.
9. Murphy M, Chatterjee SS, Jain S, Katari M, DasGupta R. TCF7L1 Modulates Colorectal Cancer Growth by Inhibiting Expression of the Tumor-Suppressor Gene EPHB3. Sci Rep. 2016 23:6:28299.

10. Eshelman MA, Shah M, Raup-Konsavage WM, Rennoll SA, Yochum GS. TCF7L 1 recruits CtBP and HDAC1 to repress DICKKOPF4 gene expression in human colorectal cancer cells. Biochem Biophys Res Commun. 2017:487(3):716-22.

11. Ma H, Mallampati S, Lu Y, Sun B, Wang E, Leng X, et al. The Sox4/Tcf7l1 axis promotes progression of BCR-ABL-positive acute lymphoblastic leukemia. Haematologica. 2014;99(10):1591-8.

12. Ku AT, Shaver TM, Rao AS, Howard JM, Rodriguez CN, Miao Q, et al. TCF7L1 promotes skin tumorigenesis independently of $\beta$-catenin through induction of LCN2. eLife. 2017;03:6.

13. Shan J, Shen J, Wu M, Zhou H, Feng J, Yao C, et al. Tcf7l1 acts as a suppressor for the self-renewal of liver Cancer stem cells and is regulated by IGF/MEK/ERK signaling independent of $\beta$-catenin. Stem Cells Dayt Ohio. 2019;37(11):1389-400.

14. Baldauf MC, Orth MF, Dallmayer M, Marchetto A, Gerke JS, Rubio RA, et al. Robust diagnosis of Ewing sarcoma by immunohistochemical detection of super-enhancer-driven EWSR1-ETS targets. Oncotarget. 2018;9(2):1587-601

15. de Graauw M, van Miltenburg MH, Schmidt MK, Pont C, Lalai R, Kartopawiro J, et al. Annexin A1 regulates TGF-beta signaling and promotes metastasis formation of basal-like breast cancer cells. Proc Natl Acad Sci U S A. 2010;107(14):6340-5.

16. Liu X, Yuan H, Zhou J, Wang Q, Qi X, Bernal C, et al. LMO7 as an unrecognized factor promoting pancreatic Cancer progression and metastasis. Front Cell Dev Biol. 2021;9:647387.

17. Ueda M, Iguchi T, Masuda T, Komatsu H, Nambara S, Sakimura S, et al. Up-regulation of SLC9A9 promotes Cancer progression and is involved in poor prognosis in colorectal Cancer. Anticancer Res. 2017 May;37(5):2255-63.

18. Wang K-Y, Huang R-Y, Tong X-Z, Zhang K-N, Liu Y-W, Zeng F, et al. Molecular and clinical characterization of TMEM71 expression at the transcriptional level in glioma. CNS Neurosci Ther. 2019;25(9):965-75.

19. Franzetti G-A, Laud-Duval K, van der Ent W, Brisac A, Irondelle M, Aubert $\mathrm{S}$, et al. Cell-to-cell heterogeneity of EWSR1-FLI1 activity determines proliferation/migration choices in Ewing sarcoma cells. Oncogene. 2017;36(25):3505-14.

20. Brabletz T, Jung A, Spaderna S, Hlubek F, Kirchner T. Opinion: migrating cancer stem cells - an integrated concept of malignant tumour progression. Nat Rev Cancer. 2005:5(9):744-9.

21. Sannino G, Marchetto A, Kirchner T, Grünewald TGP. Epithelial-to-mesenchymal and mesenchymal-to-epithelial transition in mesenchymal tumors: a paradox in sarcomas? Cancer Res. 2017;77(17):4556-61.

\section{Publisher's Note}

Springer Nature remains neutral with regard to jurisdictional claims in published maps and institutional affiliations.

Ready to submit your research? Choose BMC and benefit from

- fast, convenient online submission

- thorough peer review by experienced researchers in your field

- rapid publication on acceptance

- support for research data, including large and complex data types

- gold Open Access which fosters wider collaboration and increased citations

- maximum visibility for your research: over 100M website views per year

At BMC, research is always in progress.

Learn more biomedcentral.com/submissions 